\title{
EDITORIAL
}

\section{Surgical alternatives to CPAP in the treatment of obstructive sleep apnea}

\author{
Shirley Tarabichi $\oplus^{\circ}$, Resident Doctor \\ ENT\&HNS Department, "Sfanta Maria” Hospital, Bucharest, Romania
}

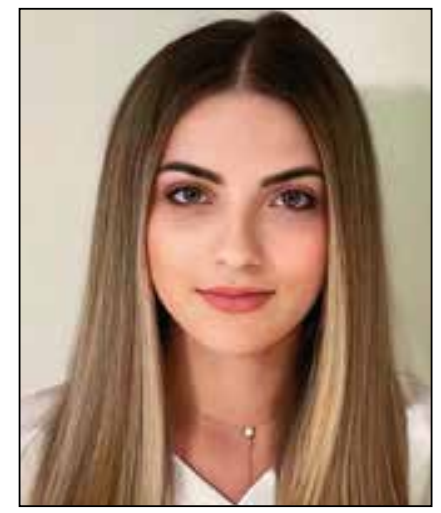

$\mathrm{T}$ he COVID-19 situation had an important outcome both on resident's education and patient's treatment, especially non-acute cases. A study made by the coordinators of the residency program at Allegheny General Hospital in Pittsburgh, Pennsylvania, presented statistics according to which more than $50 \%$ of surgery residents believe that their professional training suffered from COVID-19 pandemic crisis. Another aspect highlighted in their research indicated that $79 \%$ of them agreed that the modified program ('on' for one week, 'off' for the next) carried out by their programs of residency was a good idea to minimize exposure to direct contact with the patient even if it affected their training, clinical and operative experience ${ }^{1}$. More than that, the National level measures that were implemented regarding the decision to decrease elective procedures and the number of patients who can be hospitalized in a department, as well as the new protocols implemented lead to fewer preoperative and postoperative appointments. The most affected patients were those with chronic pathology who required a basic surgical intervention. An example among many others are the patients who are suffering from obstructive sleep apnea (OSA).

OSA is a commune and complex syndrome represented by repetitive and intermittent episodes of breathing interruption during sleep causing hypoxemia, hypercapnia, neurocognitive impairment, daytime somnolence, reduced quality of life and also increases the individual risk for developing cardiovascular disease or to emphasize the preexisting ones. Epidemiological studies estimate a prevalence of obstructive sleep apnea of $24 \%$ among men and $9 \%$ for women ${ }^{2}$.

Continuous positive airway pressure (CPAP) is considered to be the first line treatment for moderate and severe OSA cases, being intended to keep the upper respiratory tract open during sleep. However, some of the patients do not tolerate CPAP due to the fact that it can cause skin irritation, claustrophobia, or it is difficult for them to accept sleeping connected to a mechanical device. Less than $50 \%$ of patients with OSA who have been prescribed the use of CPAP have shown compliance, so its efficacy can be limited by the patient's uncertain compliance to therapy. In cases of noncompliant patients, surgical treatment may be a feasible alternative. Surgery consists of either soft tissue removal or a skeletal modification according to the site(s) of obstruction, the approach being particular for each individual.

Although CPAP opens the entire airway and surgery only targets certain specific areas, the main goals of surgical treatment are to eliminate or minimize the occurrence of respiratory collapse during sleep as well as normalization of sleep quality, improvement of the apnea-hypopnea index (a reduction of the apnea-hypopnea index by $50 \%$ ) and oxygen saturation levels. Often, a combination of procedures is necessary to achieve success ${ }^{2,3}$.

Nasal interventions are utilized to alleviate nasal obstructions caused by cartilage, bone or hypertrophic tissues by correction of a deviated nasal septum, inferior turbinates reduction or sinus surgery. The literature has shown that nasal permeabilization alone rarely cures sleep apnea, but it can enhance sleep quality by improving nasal airflow and may increase tolerance to CPAP by reducing the required pressure and the hours of use ${ }^{3,4}$.

Uvulopalatopharyngoplasty (UPPP) is, for more 
than 25 years, the most common surgical procedure for OSA due to the fact that obstructions are most frequently found in the oropharynx. This method was developed to remove the excess tissues of the lateral pharyngeal wall, hypertrophic tonsils and soft palate, in order to enlarge the pharyngeal airway. Studies found that the success rate is around $40 \%^{2}$ with UPPP surgery alone and $90 \%{ }^{4}$ of UPPP-treated patients presented a reduction in snoring events. The uvulopalatal flap (UPF) is a modified version of UPPP and has similar rates of success $^{4}$. UPF objective is to create a reversible flap; through the flap, the uvula withdraws to the pharyngeal wall reaching the velum, this resulting in the expansion of the oropharin $x^{3-5}$.

During the deep stages of sleep, when muscles are too relaxed, obstruction of the retrolingual respiratory space can occur due to the base of the tongue that falls back and comes in contact with the posterior wall of the pharynx, obstructing it. Fat deposits and lingual tonsil hypertrophy can lead to a larger tongue base size. Procedures for tongue base reduction include transoral robotic surgery base-of-tongue reduction (TORS BOT), radiofrequency ablation (RFA) and reduction glossectomy. RFA is a minimally invasive procedure that ablates tissues using low energy radio waves, while the surrounding tissues are not affected and cures obstruction in $36 \%$ of patients ${ }^{3}$, but it has great benefits in reducing snoring. Posterior midline glossectomy has a variable rate of success, ranging from 25 to $83 \%^{3}$ and consists of surgical excision of the central portion of the tongue root. TORS BOT has a success rate of approximately $68 \%{ }^{4}$ and it is a relatively new method that uses a surgical robotic device to assist the intervention that decreases the size of the tongue's root.

The genioglossus muscle is the main muscle involved in airway dilatation. In OSA, multiple studies have shown that the genioglossus muscle tone is altered $^{3-5}$. The most common procedure performed for tongue advancement is genioglossus advancement, which requires a forward moving of the genioglossus muscle to maintain the tongue base anteriorly, limiting the tongue to fall backward during sleep, thus increasing the retrolingual respiratory space and being a minimally invasive intervention. The success rate ranges from 39 to $78 \%{ }^{3}$ and, in order to maximize the result, it is generally performed with UPPP. To increase the retrolingual space, hyoid advancement can also be used and various studies have demonstrated a high rate of success ${ }^{3-5}$. The epiglottis will be advanced anteriorly as the result of this procedure and the airway will be expanded, also preventing the collapse.

As it can be seen, there are many surgical options available to treat patients with OSA which cannot tolerate CPAP therapy. Because of the anatomy of the airways, severity and due to the fact that the site of obstruction can vary significantly between individuals, the type of intervention must be tailored to each individual.

\section{REFRENCES}

1. Rana T, Hackett C, Quezada T, Chaturvedi A, Bakalov V, Leonardo $\mathrm{J}$, et al. Medicine and surgery residents' perspectives on the impact of COVID-19 on graduate medical education. Med Educ Online. 2020;25(1):1818439. DOI: 10.1080/10872981.2020.1818439.

2. Phan NT, Wallwork B, Panizza B. Surgery for adult patients with obstructive sleep apnoea: A review for general practitioners. Aust Fam Physician. 2016;45(8):574-8.

3. Carvalho B, Hsia J, Capasso R. Surgical therapy of obstructive sleep apnea: a review. Neurotherapeutics. 2012;9(4):710-6. DOI: 10.1007/ s13311-012-0141-x

4. Samuelson JC, Morrow LE, Malesker MA. Surgical procedures for obstructive sleep apnea. [Internet]. US Pharm. 2018;43(1):HS2 HS7. Available from: https://www.uspharmacist.com/article/surgical-procedures-for-obstructive-sleep-apnea.

5. Ahmed MMEDE. Single-stage drug-induced sleep endoscopy, nasal surgery and modified barbed soft palatal posterior pillar flap palatopharyngoplasty for treatment of obstructive sleep apnea. On J Otolaryngol \& Rhinol. 2020:2(2). OJOR.MS.ID.000532. DOI: 10.3352/ OJOR.2020.02.000532.

This is an open access article published under the terms and conditions of the Creative Commons Attribution-NonCommercial-NoDerivatives 4.0 International (CC BY-NC-ND 4.0) License (https://creativecommons.org/licenses/by-nc-nd/4.0/). CC BY-NC-ND 4.0 license requires that reusers give credit to the creator by citing or quoting the original work. It allows reusers to copy, share, read, download, print, redistribute the material in any medium or format, or to link to the full texts of the articles, for non-commercial purposes only. If others remix, adapt, or build upon the material, they may not distribute the modified material. 